Ann. Abeille, 1967, $10(2), 97-102$.

\title{
DER TRACHTWERT VON POLYGALA CHAMAEBUXUS
}

\author{
Annemarie FOSSEL \\ Aigen im Ennstal (Autriche)
}

SOMMAIRE

Der Zwergbuchs, Polygala chamaebuxus, ist in den Alpen auf sonnigen, trockenen Magermatten auf Kalkunterlage sehr verbreitet und seine weissgelben Blüten werden lebhaft von IIonigbienen besucht. Dennoch lassen sich bei der pollenanalytischen Untersuchung von Frühtrachthonigen keine Pollenkörner von Polygala chamaebuxus nachweisen, obwohl ihr Bau so charakteristisch und auffallend ist, dass sie kaum übersehen werden können. Der Autor beobachtete daher die Bienen beim Besuch der Blüten und stellte fest, dass die Honigbienen hier geschickt Nektardiebstahl begehen und beim Blütenbesuch garnicht mit den Staubbeuteln in Berührung kommen, so dass der eingetragene Nektar zwangsläufig pollenfrei ist.

\section{EINLEI'TUNG}

Mein Bienenstand befindet sich im steirischen Ennstal, einem von West nach Ost verlaufenden Gebirgstal, zwischen der Zentralalpenkette und den Nördlichen Kalkalpen in $680 \mathrm{~m}$ Seehöhe. Dieses Tal ist ein ausgesprochenes Frühtrachtgebiet, wo die ersten ausgiebigen Waagstockzunahmen bereits Ende April oder Anfang Mai nach wenigen vorangegangenen Flugtagen mit Pollentracht ganz unvermittelt einsetzen. Der Monat Mai ist hier die beste Trachtzeit des Bienenjahres und liefert je nach Wetterlage 50-80 p. Ioo der gesamten Honigernte.

Obst, Weiden (Salix), Bergahorn (Acer pseudoplatanus) und die ersten Frühlingsblumen bringen sonst im allgemeinen nur eine Entwicklungstracht, die die Bienenvölker erstarken lässt und dabei fast zur Gänze verbraucht wird. Im Ennstal kommt es dagegen zu Tageszunahmen bis zu $4 \mathrm{~kg}$, so dass aus der Frühtracht geschleudert werden kann.

Diese Frühtrachthonige sind pollenreiche Blütenhonige, die in ihrem Sediment bei der pollenanalytischen Untersuchung die ganze, artenreiche Flora des Bergfrühlings enthalten, die den Honigbienen Nektar liefert, nur der Zwergbuchs fehlt im Pollenbild! 
Den charakteristischen Pollen von Polygala chamaebuxus mit seinen I2I6 Iängsfalten konnte ich bisher noch in keinem Friihtrachthonig finden, obwohl die wohlriechenden Blïten von den Honigbienen sehr lebhaft beflogen werden und in grosser Menge vorkommen. Erst die Beobachtung der Sammlerinnen klärte diese Frage und machte mich auf eine gute Trachtquelle aufmerksam, von der das Pollenbild der Honige nichts verrät.

\section{SYSTEMATIK UND VERBREITUNG VON " IOLYGALA CHAMAEBUXUS"}

Polygala chamaebuxus, wegen der immergrïnen, ledrigen Blätter Zwerbuchs genannt und im Volksmund als Marillenbliimerl bezeichnet, weil es köstlich und zart nach Marillen (Aprikosen) duftet, gehört zur Familie der Polygalaceen (Kreuzblumengewächse), einer in sich geschlossenen, sehr natürlichen Pflanzenfamilie, die mit keiner anderen näher verwandt ist, was sich auch im eigenartigen Bau der Pollenkörner zeigt, die nach ZANDER (I94I), durch eine grosse Zahl von schmalen Längsfalten oder Schlitzen (9-24) gekennzeichnet sind.

Die Familie umfasst nach HeGr (I935) Io Gattungen mit ca 800 Arten, von denen die Gattung Polygala mit über $45^{\circ}$ Arten die grösste und zugleich kosmopolitisch ist. Die Sektion Chamaebuxus mit ca 23 Arten ist hauptsächlich in der Nordhemisphäre verbreitet (America, Asien, E,uropa), reicht aber auch bis ins Kapland von Afrika.

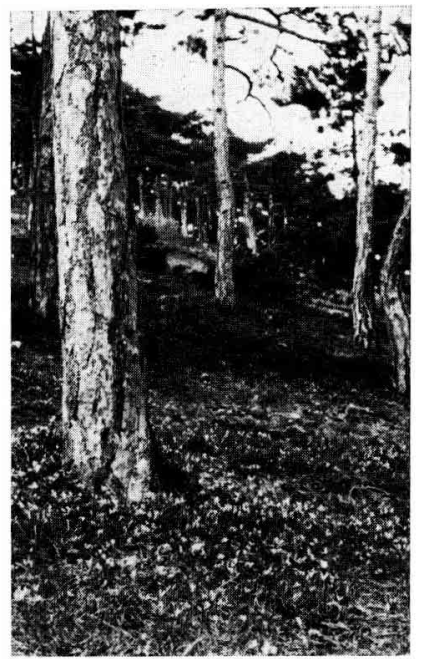

Aвв :. -.. J'olygala chanaebuxus in Schioraförenvald (Pinus austriaca) ion Nieder-Osterreich fic. I. - Polygala chamaebuxus dans la forit de pins noirs (Pinus austriara) de Basse Autriche

Meine Beobachtungen beziehen sich nur auf Polygala chamaebuxus.

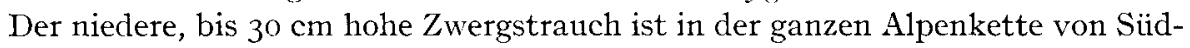
frankreich bis zu den Ostalpen und in den Illyrischen Gebirgen beheimatet und gehört zum mediterran-präalpinen Element oder zur pontischen Steppengemeinschaft.

Die Pflanze ist ein reichverzweigter Halbstrauch, der mit Vorliebe auf Kalk gedeiht, aber auch auf Granit, Gneis, Porphyr und Glimmerschiefer dichte Teppiche 
bildet, die in Gesellschaft von Erica carnea oder Calluna vulgaris, Vaccinium sp., Helianthemum, Thymus usw., gute Trachtgebiete darstellen.

In Osterreich ist das Marillenblümerl besonders auf Kalk von der Ebene bis in die untere alpine Stufe stellenweise stark verbreitet, im Wallis (Schweiz) steigt es sogar bis $2480 \mathrm{~m}$. $A b b .1$ zeigt blühenden $Z$ wergbuchs in einem Schwarzföhrenwald (Pinus austriaca) in Nieder-Osterreich und veranschaulicht, wie dicht und ausgedehnt diese Bestände sind.

\section{BLÜTENBAU UND BESTÄUBUNGSVERHÄLTNISSE}

Die Bliitenknospen von Polygala chamaebuxus werden schon im Herbst angelegt und treten voll entwickelt in die Winterruhe ein, bedürfen aber in den Alpen des Schutzes einer winterlichen Schneedecke.

Die weisslichgelben, etwa $5_{5} \mathrm{~mm}$ langen Blüten, die sich im zeitigen Friihjahr öffnen, besitzen 5 Kelch- und 5 Kronblätter von ungleicher Gestalt.

Das obere Kelchblatt ist blassgriin, klein und hochgewölbt oder wie eine Kappe geformt und verdeckt den Fingang zum Nektar. Die beiden seitlichen Kelchblätter sind gross, länglich-spatelförmig und weisslich, wie Kronblätter und bilden zwei aufgerichtete oder zuriickgeschlagene Fahnen. Auf der Unterseite befinden sich die beiden restlichen Kelchblätter, die klein, schmal und blassgrün sind. Die zwei Fahnen färben sich nach der Blüte braunrot oder purpurn.

Die 5 Kronblätter schmiegen sich dicht aneinander und bilden einen seitlich zusammengedrückten Behälter, eine Art Schiffchen, das sich nur nach oben öfnet und die 8-Io Staubblätter und den oberständigen Fruchtknoten samt Griffel und Narbe umschliesst. Die seitlichen Kronblätter sind gelb oder weisslich, das vordere ist zitronengelb bis orangerot und färbt sich nach der Bliite purpurn bis bräunlich.

Die Blüten von Polygala chamaebuxus sind proterandrisch, die dreifächrigen Staubbeutel lassen ihre drei Klappen schon in der Blïtenknospe aufspringen und entleeren den gelben, stark ölhaltigen Pollen in den Vorderteil des von den Kronblättern gebildeten Behälters.

Als Bestäuber kommen vor allem Hummeln in Frage, die sich auf dem waagrecht stehenden Schiffchen niederlassen und es herabdrücken, wobei ihnen ein Teil des Pollens, der sich im Behälter befindet, an den Bauch gedrückt wird. Auch die Narbe tritt hervor, berührt den Insektenleib und kann so mit Blütenstaub belegt werden. Selbstbestäubung führt nicht zur Fruchtbildung.

Der Nektar wird am Blütengrunde von einer Drüse abgesondert, die aus dem reduzierten Diskus hervorgegangen ist. Die Nektarproduktion ist so reichlich, dass man den Tropfen mit freiem Auge erkennen kann, wenn man das obere Deckblatt abhebt.

\section{INSEKTENBESUCH UND TRACH'TWERT}

In meinem Beobachtungsgebiet wird der Zwergbuchs vor allem von Honigbienen und räuberischen Hummeln besucht. Stellenweise werden über 90 p. Ioo der Blïten von Hummeln ausgeraubt. 
Die Hummeln beissen entweder ein Loch in das obere Kelchblatt $(A b b .2)$ oder sie zwicken die Spitze des Kelchblattes einfach ab und gelangen so mit ihrem Riissel zum Nektar. Sie sind dabei so flink und geschickt, dass man zunächst kaum bemerkt, was hier vorgeht. Eine Hummel soll nach HEGI (I935) in 5 Minuten 40 Löcher beissen können!

\section{Blütenbau V. Polygala chamaebuxus}
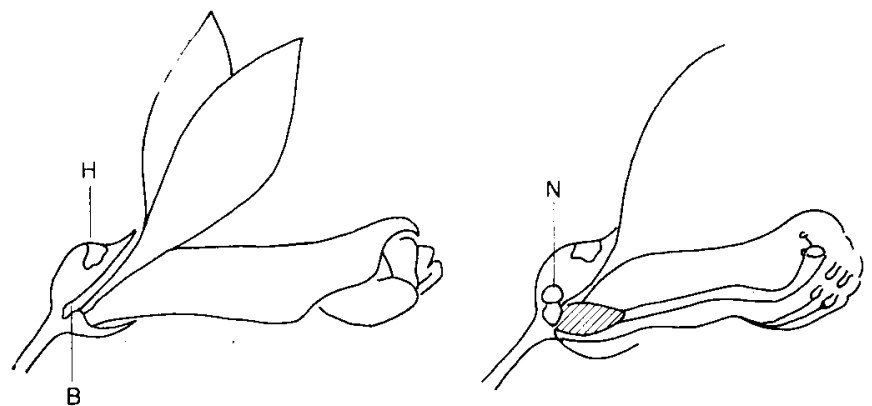

H-Hummel, B-Biene, N-Nektor

$\mathrm{AEB} .2 .--$ Skizze der Blütenbaues:

H. Hummeleinbiss, B. Saugstelle der Bienen, N-Nektartropfen

FIc. 2. - Schéma de la fleur

H. Point que déchire le Bourdon. B. Emplacement où les abeilles introduisent leur trompe, N. goutte de nectar

Ein Teil der Honigbienen benützt die Einbruchslöcher der Hummeln, um Nektar zu sammeln. Sie bilden eine gut eingeflogene Diebsbande, die aus den grossen Bisslöchern der räuberischen Hummeln mühelos Nutzen zu ziehen weiss.

$A b b .3$ zeigt uns eine solche Biene und wir erkennen deutlich, dass sie sich seitlich des Schiffchens niedergelassen hat und mit den von den Kronblättern um schlossenen Staubbeuteln und der Narbe garnicht in Berührung kommt.

Der Bau und die Stellung der Blüten verhindern auch, dass der Blütenstaub, der ganz vorn im Schiffchen deponiert ist, durch Zufall zum Nektar gelangt, denn die Nektardriise befindet sich hinter dem Fruchtknoten, wie die Skizze auf $A b b .2$ zeigt.

Eine grosse Zahl von Honigbienen ist aber nicht von den Bisslöchern der Hummeln abhängig, sondern benützt einen andern Weg. Diese Diebe fliegen die Marillenblümerln ganz seitlich an und zwängen ihren Rïssel am Ansatz der Blïte unter das obere Kelchblatt und erreichen auf diese Weise den Nektar. Nach der grossen Zahl der sammelnden Honigbienen zu schliessen, ist diese Nektarquelle sehr beliebt. Man findet aber stets nur diebische Nektarsammlerinnen, nie eine Biene mit Pollenhöschen, so dass die Honigbiene nach meinen Beobachtungen nicht zu den Bestäuberinnen von Polygala chamaebuxus zu zählen ist.

Frischt man einige bliihende Zweige von Zwergbuchs zu Hause in Wasser ein, so zeigt sich bald, dass der Nektar so reichlich abgesondert wird, dass er an der Stelle, an der die Bienen saugen, zwischen den Kelchblättern austritt. Ich halte daher den $Z$ wergbuchs für eine sehr gute Trachtquelle, die gewiss überall dort, wo die 
Pflanze in grossen Mengen gedeiht eine bienenwirtschaftlich nicht umbedeutende Rolle spielt.

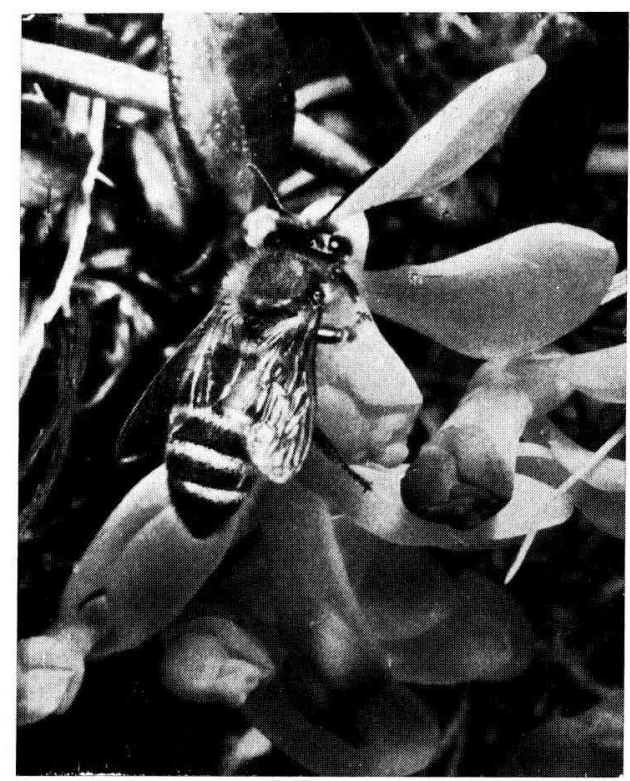

ABr. 3. - Honigbiene bein Nektardiebstahl auf Polygala chamaebuxus

Fig. 3. - Abeille suçant du nectar sur Polygala chamaebuxus

\section{SCHLUSSFOLGERUNG}

Um das 'Trachtangebot im Flugkreis bestimmter Bienenvölker genau kennen zu lernen ist, wie obiges Beispiel zeigt, neben der pollenanalytischen Untersuchung von zahlreichen Honigproben auch eine Kontrolle der verbreitetsten Trachtpflanzen, die Kenntnis ihres Blïtenbaues und die Beobachtung des Bienenbesuches sehr wichtig.

Beim Besuch von Polygala chamaebuxus erweisen sich die Honigbienen als sehr geschickte Nektardiebe, die in grosser Zahl eine sehr verbreitete Nektarquelle ausbeuten, ohne dass sich diese Tracht im Pollenbild der geernteten Honige bemerkbar macht. Bei den ersten Frühjahrsblumen ist dies zwar bei Tröpferltracht leicht möglich, weil der grösste Teil des Sammelgutes bei der stürmischen Entwicklung der Bienenvölker sofort wieder verbraucht wird, aber in ausgesprochenen Frühtrachtgebieten, wie z. B. im İnnstal, pflegt die ganze Skala der ersten Nektarspender im Pollenbild vertreten zu sein.

Der Bliutenbau und das Verhalten der Honigbienen erklären, wieso beim Besuch von Polygala chamaebuxus iiberhaupt kein Pollen in den Honigmagen der Nektarsammlerinnen gelangt. 
Diese Feststellung schmälert in keiner weise die vielseitige Aussagskraft der heute bereits altbewährten Pollenanalyse, sondern beweist nur, dass die Trachtquellen der Bienen und die Geschicklichkeit der kleinen Sammlerinnen noch immer nicht bis in alle Einzelheiten bekannt sind.

Reçu pour publication en juin 1967.

\section{RÉSUMÉ}

\section{LA RÉCOLTE DU POLLEN ET DU NECTAR SUR " POLYGALA CHAMAEBUXUS "}

Le buis nain, Polygala chamaebuxus, est très répandu dans les Alpes, dans les prairies maigres, sèches et ensoleillées, sur les sols calcaires ; ses fleurs jaune clair sont activement visitées par les Abeilles. Cependant l'analyse pollinique de miels provenant d'une miellée précoce ne montre aucun pollen de Polygala chamaebuxus alors que sa morphologie est si caractéristique et si frappante qu'il est difficile de ne pas le voir. C'est pourquoi l'auteur a observé le butinage des abeilles et a constaté qu'elles dérobent adroitement le nectar sans entrer en contact avec les anthères des fleurs visitées si bien que dans le nectar ainsi obtenu il n'y a absolument pas de pollen.

\section{SUMMARY}

THE POLLEN AND NECTAR HARVEST OF " POLYGALA CHAMAEBUXUS"

The dwarf box (Polygala chamaehuxus) is very widespread in the Alps in poor dry and sunny meadows on calcareous soils; its bright yellow flowers are actively visited by bees. However, pollen analysis of honeys from an early honey-flow shows no pollen from Polygala chamaebuxus. For this reason the author observed the honey-gathering of bees on these fowers and established that they remove the nectar so skilfully that they do not come into contact with the anthers.

\section{ZUSAMMENFASSUNG}

Die niedere, dichtverzweigte Polygala chamaebuxus ist in den Alpen von der Ebene bis in die untere alpine Stufe auf sonnigen Magermatten stark verbreitet und bildet in Gesellschaft von Erica carnea oft ausgedehnte Bestände, die schon im ersten Frühjar blühen. Die nektarreichen, zart nach Marillen duftenden Blüten locken neben räuberischen Hummeln auch zahlreiche Honigbienen an, die jedoch die Bestäubung nicht vollziehen sondern geschickt Nektardiebstahl begehen, indem sie entweder die Einbruchslöcher der Hummeln benützen oder seitlich zwischen den Kelchblättern einen illegalen Zugang zum Nektar finden.

Durch diese Art des Blütenbesuches und den besonderen Bau des Zwergbuchses gelangt kein Pollen in den Ncktar oder in den Honigmagen der Sammlerinnen, so dass diese Trachtquelle im mikroskopishcen Pollenbild der Honige nicht nachzuweisen ist, obwohl sie stellenweise bienenwirtschaftlich eine nicht unbedeutende Rolle spielen dürfte.

\section{I,ITERATUR}

HegI G., I935. Illustrierle Flora Milteleuropas, Bd 5-I, 86-95, Karl Hanser-Verlag München. ZaNDER E., r941. Beiträge zur Herkunftsbestimmung bei Honig, Bd. 3, 48-5o, Verlag Liedloff, Leipzig. 